\title{
ON THE LOWER ORDER OF INTEGRAL FUNCTIONS
}

\section{S. M. SHAH}

Let $f(z)=\sum_{0}^{\infty} a_{n} z^{n}$ be an integral function of order $\rho$. It is known that $^{1}$

(1) $\limsup _{n \rightarrow \infty} \frac{n \log n}{\log \left\{1 /\left|a_{n}\right|\right\}}=\rho=\limsup _{r \rightarrow \infty} \frac{\log \log M(r)}{\log r}(0 \leqq \rho \leqq \infty)$.

A similar result for the lower ${ }^{2}$ order $\lambda$, namely

$$
\liminf _{n \rightarrow \infty} \frac{n \log n}{\log \left\{1 /\left|a_{n}\right|\right\}}=\lambda=\liminf _{r \rightarrow \infty} \frac{\log \log M(r)}{\log r},
$$

does not always hold. In fact for

$$
\begin{gathered}
\exp \left(z^{2}\right)+\exp (z)=2+z+z^{2}\left(\frac{1}{1 !}+\frac{1}{2 !}\right)+\cdots, \\
\liminf _{n \rightarrow \infty} \frac{n \log n}{\log \left\{1 /\left|a_{n}\right|\right\}}=1
\end{gathered}
$$

whereas $\lambda=\rho=2$.

We prove here the following theorem.

THEOREM 1. If $f(z)=\sum_{0}^{\infty} a_{n} z^{n}$ is an integral function of order $\rho$ and lower order $\lambda(0 \leqq \lambda \leqq \infty)$ then

$$
\lambda \geqq \liminf _{n \rightarrow \infty} \frac{n \log n}{\log \left\{1 /\left|a_{n}\right|\right\}} \geqq \liminf _{n \rightarrow \infty} \frac{\log n}{\log \left|a_{n} / a_{n+1}\right|} .
$$

\section{COROLLARY $1 .^{3}$}

$$
\begin{gathered}
\liminf _{n \rightarrow \infty} \frac{\log \left|a_{n} / a_{n+1}\right|}{\log n} \leqq \liminf _{n \rightarrow \infty} \frac{\log \left\{1 /\left|a_{n}\right|\right\}}{n \log n}=\frac{1}{\rho} \leqq \frac{1}{\lambda} \\
\leqq \limsup _{n \rightarrow \infty} \frac{\log \left\{1 /\left|a_{n}\right|\right\}}{n \log n} ; \leqq \limsup \frac{\log \left|a_{n} / a_{n+1}\right|}{\log n}
\end{gathered}
$$

Received by the editors April 4, 1946, and, in revised form, May 3, 1946.

${ }^{1}$ E. C. Titchmarsh, Theory of functions, pp. 253-254; E. T. Copson, Theory of functions of a complex variable, pp. 175-178.

2 For the definition, and so on, see (i) J. M. Whittaker, The lower order of integral functions, J. London Math. Soc. vol. 8 (1933) pp. 20-27; (ii) S. M. Shah, The lower order of the zeros of an integral function (II), Proceedings of the Indian Academy of Sciences (A) vol. 21 (1945) pp. 162-164.

3 Cf. a similar result (1) in S. M. Shah, The maximum term of an entire series, Mathematics Student vol. 10 (1942) pp. 80-82. 
CoRollary 2. If $\lim _{n \rightarrow \infty} n \log n / \log \left\{1 /\left|a_{n}\right|\right\}=L$ where $0 \leqq L<\infty$ then $f(z)=\sum_{0}^{\infty} a_{n} z^{n}$ is an integral function of regular growth ${ }^{4}$ and of $\operatorname{order} L$.

THEOREM 2. If (i) $f(z)=\sum_{0}^{\infty} a_{n} z^{n}$ is an integral function of order $\rho$ and lower order $\lambda(0 \leqq \lambda \leqq \infty)$ such that (ii) $\left|a_{n} / a_{n+1}\right|$ is a nondecreasing function of $n$ for $n>n_{0}$, then

$$
\begin{aligned}
& \lambda=\liminf _{n \rightarrow \infty} \frac{n \log n}{\log \left\{1 /\left|a_{n}\right|\right\}}=\liminf _{n \rightarrow \infty} \frac{\log n}{\log \left|a_{n} / a_{n+1}\right|}, \\
& \rho=\limsup _{n \rightarrow \infty} \frac{\log n}{\log \left|a_{n} / a_{n+1}\right|} .
\end{aligned}
$$

We note that the hypothesis (ii) of Theorem 2 does not imply that $f(z)$ is of regular growth. In fact we have the following theorem.

THEOREM 3. There exists an integral function $f(z)=\sum_{0}^{\infty} a_{n} z^{n}$ for which (i) $a_{n}>0$, (ii) $a_{n} / a_{n+1}$ is a steadily increasing function of $n$, and (iii) $\rho>\lambda$.

An interesting application of these results can be made to the series $F(z)=\sum_{0}^{\infty} a_{n} \epsilon_{n} z^{n}$ where $\left\{\epsilon_{n}\right\}$ are a set of numbers such that $\left|\epsilon_{n}\right|=1$ or 0 and such that $\sum_{0}^{\infty} a_{n} \epsilon_{n} z^{n}$ consists of an infinite number of terms. $F(z)$ is an integral function. Let its order be $\rho(F)$ and lower order be $\lambda(F)$. Since

$$
M(r, f) \geqq\left|a_{n}\right| r^{n} \geqq\left|a_{n} \epsilon_{n}\right| r^{n}
$$

for every $n$ and $r$, and so if $\mu(r)$ denotes the maximum term, $M(r, f)$ $\geqq \mu(r, F)$. Hence

$$
\lambda(f) \geqq \lambda(F) ; \quad \rho(f) \geqq \rho(F) .
$$

If $\left|a_{n} / a_{n+1}\right|=\psi(n)$ (say) is a nondecreasing function of $n$ then

(7) $\lambda(f)=\liminf _{n \rightarrow \infty} \frac{n \log n}{\log \left\{1 /\left|a_{n}\right|\right\}} \leqq \limsup _{n \rightarrow \infty} \frac{n \log n}{\log \left\{1 /\left|a_{n} \epsilon_{n}\right|\right\}}=\rho(F)$

and so we have the following theorem.

THEOREM 4. If $f(z)=\sum_{0}^{\infty} a_{n} z^{n}$ is an integral function of order $\rho$ and of lower order $\lambda$ and is such that $\left|a_{n} / a_{n+1}\right|$ is a nondecreasing function of $n$ for $n>n_{0}$, then $F(z)=\sum_{0}^{\infty} a_{n} \epsilon_{n} z^{n}$ is of order $\rho(F) \geqq \lambda$.

For instance every function $F=\sum_{0}^{\infty} \epsilon_{n} z^{n} / n$ ! is of order 1 .

An example, to illustrate the point that by an appropriate choice

${ }^{4}$ Cf. G. Valiron, Lectures on the general theory of integral functions, pp. 41-44. 
of $\epsilon_{n}$ the order $\rho(F)$ of $F(z)=\sum a_{n} \epsilon_{n} z^{n}$ can be made equal to any number $x$ where $\lambda(f) \leqq x \leqq \rho(f)$, is given in the proof of Theorem 3 .

The function $\exp z=\sum_{0}^{\infty} z^{n} / n !$ for which $\psi(n)$ is an increasing function of $n$ is bounded on the real negative axis and the series

$$
F(z)=z-\frac{z^{3}}{3 !}+\frac{z^{5}}{5 !}-\cdots
$$

is bounded on the real axis. If $\psi(n)$ is increasing sufficiently rapidly then we prove that $f(z)$ and $F(z)$ are not bounded on any line arg $z=\alpha$ $(0 \leqq \alpha \leqq 2 \pi)$. In fact we have the following theorem.

THEOREM 5. If $f(z)=\sum_{0}^{\infty} a_{n} z^{n}$ is an integral function of lower order $\lambda$ such that $\left|a_{n} / a_{n+1}\right| \geqq \vartheta^{2}\left|a_{n-1} / a_{n}\right|$ for $n>n_{0}$ then

(8) $\quad \limsup _{r \rightarrow \infty} \frac{\log \log m(r, f)}{\log r} \geqq \lambda ; \quad \limsup \frac{\log \log m(r, F)}{\log r} \geqq \lambda$

where $m(r, f)=\min _{|z|=r}|f(z)|$ and $\vartheta=2 \cdot 2$.

LEMMA. $a_{n}$ is any sequence of real or complex numbers such that ${ }^{5}$

$$
\left|a_{n}\right|<1 \quad \text { for } n>n_{0} \text {. }
$$

Let

$$
\begin{aligned}
\theta(n) & =\frac{\log \left\{1 /\left|a_{n}\right|\right\}}{n \log n} ; & \phi(n) & =\frac{\log \left|a_{n} / a_{n+1}\right|}{\log n} ; \\
\alpha & =\liminf _{n \rightarrow \infty} \phi(n) ; & \gamma & =\liminf _{n \rightarrow \infty}\{1 / \phi(n)\} ; \\
\beta & =\limsup _{n \rightarrow \infty} \phi(n) ; & \delta & =\limsup _{n \rightarrow \infty}\{1 / \phi(n)\} ; \\
A & =\liminf _{n \rightarrow \infty} \theta(n) ; & C & =\liminf _{n \rightarrow \infty}\{1 / \theta(n)\} ; \\
B & =\limsup _{n \rightarrow \infty} \theta(n) ; & D & =\limsup _{n \rightarrow \infty}\{1 / \theta(n)\} ;
\end{aligned}
$$

then

$$
\alpha \leqq A=1 / D ; \quad 1 / C=B \leqq \beta ; \quad C \geqq \gamma .
$$

(ii) If further $\psi(n)$ is a nondecreasing function of $n$ for $n \geqq N$ and $\psi(N) \geqq 1$ then

$$
C=\gamma=1 / \beta ; \quad D=\delta=1 / \alpha .
$$

The proof of (9) is straightforward and omitted.

\footnotetext{
- Some of the relations in (9) and (10) hold under less restrictive conditions.
} 
Proof of (10). By hypothesis (ii), $\alpha, \beta, \gamma$ and $\delta$ are non-negative and $\beta=1 / \gamma, \alpha=1 / \delta$. We prove $B \geqq \beta$. Suppose first $0<\beta<\infty$. Then

$$
\psi(n)>n^{\beta-c} \quad \text { for } n=N_{1}, N_{2}, \cdots, N_{p}, \cdots .
$$

Let $N_{1}>\max \left\{n_{0}, N\right\}$. Then

$$
\begin{aligned}
\left|\frac{1}{a_{n}}\right| & =k\left(N_{1}\right) \psi\left(N_{1}+1\right) \cdots \psi(n-1), \\
\theta(n) & =o(1)+\frac{\log \psi\left(N_{1}+1\right)+\cdots+\log \psi(n-1)}{n \log n} .
\end{aligned}
$$

Let $n=\left[N_{p} \log ^{2} N_{p}\right]+1$. Then

$$
\theta(n) \geqq o(1)+\frac{\left(n-N_{p}\right) \log N_{p}^{\beta-\epsilon}}{n \log n} .
$$

Hence $B \geqq \beta$ which holds also when $\beta=0$. If $\beta$ be infinite the above argument with an arbitrary large number instead of $\beta-\epsilon$ gives that $B=\infty$. Hence from (9) we get that $B=\beta$ and so $C=\gamma=1 / \beta$. The second relation in (10) follows similarly.

Proof of THEOREM 1. Since $\sum a_{n}$ is convergent, $\left|a_{n}\right|<1$ for $n>n_{0}$. As $C \geqq \gamma$ we need prove $\lambda \geqq C$ only. Suppose first $0<C<\infty$. Then

$$
\begin{aligned}
\frac{n \log n}{\log \left\{1 /\left|a_{n}\right|\right\}} & >C-\epsilon, \\
\left|a_{n}\right| & >n^{-n /(C-\epsilon)}, \quad \text { for all } n \geqq N(\epsilon) .
\end{aligned}
$$

Let $r_{n}=2 n^{1 /(C-\bullet)}$. If $r_{n} \leqq r \leqq r_{n+1}(n>N)$ then

$$
M(r) \geqq\left|a_{n}\right| r^{n} \geqq\left|a_{n}\right| r_{n}^{n}>n^{-n /(C-\epsilon)} \exp \left(n \log r_{n}\right)=\exp (n \log 2) .
$$

Hence $\log M(r) \geqq \log 2\left\{(r / 2)^{c-\epsilon}-1\right\}$ for all large $r$ and so $\lambda \geqq C$, which holds when $C=0$. If $C=\infty$, the above argument shows that $\lambda=\infty$.

Corollary 1 follows from (1), (2) and (9), and Corollary 2 from (1) and (2). The example given at the beginning of the paper shows that $f(z)$ may be of regular growth and $\lim _{n \rightarrow \infty}\left\{n \log n / \log \left\{1 /\left|a_{n}\right|\right\}\right\}$ may not exist.

Proof of Theorem 2. Let $\mu(r)$ denote the maximum term, $\nu(r)$ its rank. By hypothesis (ii), $\psi(n)>\psi(n-1)$ for an infinity of $n$; for if otherwise $\psi(n)=\psi(n+1)=\cdots$ ad inf for $n>p$, say, and hence the radius of convergence of the series $\sum a_{n} z^{n}$ would be finite. $\psi(n)$ tends to infinity with $n$.

When $\psi(n)>\psi(n-1)$ the term $a_{n} z^{n}$ becomes a maximum term 
and we have $\mu(r)=\left|a_{n}\right| r^{n}, \nu(r)=n$ for $\psi(n-1) \leqq r<\psi(n)$. Now $\lambda=\lim \inf _{r \rightarrow \infty} \log \nu(r) / \log r$. Suppose first that $0<\lambda<\infty$. Then $\nu(r)$ $>r^{\lambda-\epsilon}$ for $r>R=R(\epsilon)$. Let $|z|=r>R$ and let $a_{m_{1}} z^{m_{1}}$ and $a_{m_{2}} z^{m_{2}}$ $\left(m_{1}>n_{0} ; \psi\left(m_{1}-1\right)>R\right)$ be two consecutive terms so that $m_{1} \leqq m_{2}-1$ and let $m_{1}<n \leqq m_{2}$. Since $a_{m_{1}} z^{m_{1}}$ is maximum term we have $\nu(r)=m_{1}$ for $\psi\left(m_{1}-1\right) \leqq r<\psi\left(m_{1}\right)$. Hence for every $r$ in this interval $m_{1}=\nu(r)$ $>r^{\lambda-\epsilon}$. In particular $m_{1}>\left\{\psi\left(m_{1}\right)-C\right\}^{\lambda-\epsilon}$ where $C=\min \left\{1,\left(\left(\psi\left(m_{1}\right)\right.\right.\right.$ $\left.\left.-\psi\left(m_{1}-1\right)\right) / 2\right\}$. Further we have

$$
\psi\left(m_{1}\right)=\psi\left(1+m_{1}\right)=\cdots=\psi(n-1) .
$$

Hence

$$
\begin{aligned}
\psi\left(n_{0}+1\right) \cdots \psi(n-1) & =\left|\frac{a_{n_{0}+1}}{a_{n}}\right| \leqq\{\psi(n-1)\}^{n-n_{0}-1} \\
& <\left\{C+m_{1}^{1 /(\lambda-\epsilon)}\right\}^{n-n_{0}-1} \\
& <K\left(n_{0}\right) 2^{n} n^{\left(n-n_{0}-1\right) /(\lambda-\epsilon)} .
\end{aligned}
$$

Hence for all large $n$

and so

$$
\left|\frac{1}{a_{n}}\right|<K_{1}\left(n_{0}\right) 2^{n} \cdot n^{\left(n-n_{0}-1\right) /(\lambda-\epsilon)}
$$

$$
C \geqq \lambda
$$

which holds when $\lambda=0$. If $\lambda=\infty$ the above argument gives $C=\infty$. Hence from (2), $\lambda=C$ and so from (10) we get (4); and from (1) and (10) we have (5).

Proof of Theorem 3. Let $n_{1}=2, n_{s+1}=n_{s}^{4}(s=1,2,3, \cdots)$,

$$
\begin{array}{rlrl}
r_{1}=1, & r_{m}=m & \text { for } n_{s} \leqq m<n_{s}^{2}, \\
r_{m}=n_{s+1}-\frac{n_{s+1}-m}{\left\{\left(n_{s+1}\right) !\left(_{s+1}\right) !\right.} & \text { for } n_{s}^{2} \leqq m<n_{s+1},
\end{array}
$$

$s=1,2,3, \cdots$, and let

$$
f(z)=1+\sum_{1}^{\infty} \frac{z^{n}}{r_{1} r_{2} \cdots r_{n}} .
$$

Then $a_{n}>0$ and $a_{n} / a_{n+1}=r_{n+1}$ which is a steadily increasing function of $n$. Also

Hence

$$
\theta(n)=\frac{\log r_{1}+\cdots+\log r_{n}}{n \log n} .
$$




$$
\begin{gathered}
\theta\left(n_{s+1}\right) \sim \frac{\left(n_{s}^{4}-n_{s}^{2}\right) \log \left(n_{s}^{4}\right)}{4 n_{s}^{4} \log n_{s}} \sim 1, \\
\theta\left(\left[n_{s}^{2} \log n_{s}\right]\right) \sim \frac{\left(n_{s}^{2} \log n_{s}-n_{s}^{2}\right) \log \left(n_{s}^{4}\right)+O\left(n_{s}^{2} \log n_{s}\right)}{n_{s}^{2} \log n_{s}^{4} \log \left\{n_{s}^{2} \log n_{s}\right\}} \sim 2 .
\end{gathered}
$$

It is easily seen that $\lim \sup _{n \rightarrow \infty} \theta(n)=2 ; \lim \inf _{n \rightarrow \infty} \theta(n)=1$. Hence $f(z)$ is an integral function of order 1 and lower order $1 / 2$. Let now

$$
\epsilon_{m}= \begin{cases}1 & \text { when } m=\left[n_{s}^{2} \log n_{s}\right] \quad(s=1,2,3, \cdots) \\ 0 & \text { otherwise. }\end{cases}
$$

Then

$$
F(z)=\sum_{1}^{\infty} a_{n} \epsilon_{n} z^{n}=\sum_{1}^{\infty} \frac{\epsilon_{n} z^{n}}{r_{1} r_{2} \cdots r_{n}}
$$

is an integral function of order $1 / 2$. If

$$
\epsilon_{m}=\left\{\begin{array}{ll}
1 & \text { when } m=n_{s} \\
0 & \text { otherwise }
\end{array} \quad(s=1,2,3, \cdots)\right.
$$

then $F(z)$ is of order 1 . Let $1 / 2<x<1$ and $\epsilon_{m}=1$ when $m=\left[\exp \left(4 x \log n_{s}\right)\right]$ $(s=1,2,3, \cdots)$ and zero otherwise; then $F(z)$ is of order $x$.

Proof of Theorem 5. Let $\left|\epsilon_{n}\right|=1$ for $n=N_{1}, N_{2}, \cdots N_{p}, \cdots$ $\left(N_{1}>n_{0}\right)$. We write $N_{p}=N$. Let $R_{N}=\vartheta \psi(N-1)$ and $|z|=R_{N}=R$.

$$
\mu(r, f)=\left|a_{N}\right| r^{N}=\mu(r, F) \quad \text { for } \psi(N-1) \leqq r<\psi(N)
$$

and $R$ lies inside this interval.

Now

$$
\begin{aligned}
|f(z)| & =\left|\sum_{0}^{N-1} a_{n} z^{n}+a_{N} z^{N}+\sum_{N+1}^{\infty} a_{n} z^{n}\right| \\
& \geqq \mu(R, f)-\left|\sum_{0}^{N-1} a_{n} z^{n}\right|-\left|\sum_{N+1}^{\infty} a_{n} z^{n}\right| .
\end{aligned}
$$

$$
\begin{aligned}
\left|\sum_{0}^{N-1} a_{n} z^{n}\right| & \leqq\left|a_{N-1}\right| R^{N-1}+\cdots \\
& \leqq \mu(R)\left\{\frac{1}{\vartheta}+\frac{1}{\vartheta^{4}}+\frac{1}{\vartheta^{9}}+\cdots+\frac{1}{\vartheta^{\left(N-n_{0}-2\right)^{2}}}+c(1)\right\} \\
& \leqq \mu(R)\left\{\frac{1}{\vartheta}+\frac{1}{\vartheta^{4}}+\frac{1}{\vartheta^{9}}+\cdots \operatorname{adinf}\right\}+\frac{\mu(R)}{10^{10}}
\end{aligned}
$$


for all large $N$.

$$
\begin{aligned}
\left|\sum_{N+1}^{\infty} a_{n} z^{n}\right| & \leqq\left|a_{N+1}\right| R^{N+1}+\cdots \\
& \leqq \mu(R)\left\{\frac{1}{\theta}+\frac{1}{\theta^{4}}+\frac{1}{\theta^{9}}+\cdots\right\} .
\end{aligned}
$$

Hence for all large $R$

$$
|f(z)|>\frac{\mu(R, f)}{10000} .
$$

Similarly

$$
|F(z)|>\frac{\mu(R, f)}{10000} .
$$

Hence $f$ and $F$ are not bounded on any line $\arg z=\alpha$.

Since

$$
\liminf _{r \rightarrow \infty} \frac{\log \log \mu(r, f)}{\log r}=\lambda
$$

the theorem follows.

Added in proof. A short note containing a part of each of the Theorems 1, 2, and 3 appeared in J. Indian Math. Soc. vol. 9 (1945) pp. 50-54.

MUSLIM UNIVERSITY 